\title{
A systematic review of randomised controlled trials examining Curcuma longa for dementia
}

\begin{abstract}
Curcuma longa has been used to treat several ailments traditionally, such as rheumatoid arthritis, conjunctivitis, skin cancer, small pox, chicken pox, wound healing, urinary tract infections and liver ailments. Due to its therapeutic properties, it has recently grown in popularity as a potential adjunct therapy for dementia. However, no systematic review of randomised controlled trials has been conducted to evaluate its efficacy and safety for cognitive decline. In this dissertation, literature searches were conducted using four electronic databases, including PubMed Central, Science Direct, CINAHL, and PsycINFO. We retrieved five published studies which were randomised control trials and have included these in the review. Search terms used were 'dementia', 'Alzheimer*', 'ageing', 'mild cognitive impairment', 'cognitive decline', 'turmeric' and 'curcuma longa'. Efficacy measures such as biochemical markers, neuropsychological assessments, and pharmacological measures have been extracted from studies and synthesised in the review. Although several in vitro and in vivo model studies have found neuroprotective effects of curcumin in Alzheimer's disease, there is not sufficient evidence to support curcumin use in humans with Alzheimer's disease, and healthy volunteers. Short term usage of curcumin has been found to be safe. Various reasons such as its low bioavailability and inconsistency in study designs were responsible for discrepancies between preclinical findings and human trials.
\end{abstract}

Keywords: dementia, alzheimer's disease, ageing, mild cognitive impairment, cognitive decline, turmeric, curcuma longa
Volume I 3 Issue 6 - 2020

\author{
Shivani P Shah, Genevieve Z. Steiner \\ 'NICM,Western Sydney University, Penrith NSW 275I, Australia
}

Correspondence: Shivani P Shah, Western Sydney University, Locked Bag 1797, Penrith NSW 275I, USA,

Email18263389@student.westernsydney.edu.au

Received: September 21, 2020 | Published: December 31, 2020

\section{Introduction}

\section{Turmeric and its applications}

Turmeric is yellow coloured spice derived from rhizomes of Curcuma longa, which belongs to the ginger family (Zingiberacea). Curcumin, which is diarylheptanoid polyphenol; is the major active constituent of turmeric, which contains more than 28 phytocompounds (Veniglla, Gyengesi, \& Muench, 2015). Traditional use of turmeric is as a culinary spice; it is also prescribed as an herbal medicine in traditional medicine systems. For example, turmeric is well known for its body cleansing properties in Ayurvedic, Chinese, and Persian medicine. It is also used in Indian, Asian, and Middle Eastern foods. ${ }^{1}$ Traditionally, in Ayurvedic medicine, turmeric has been used to treat ailments such as arthritis, gastric ulcers, jaundice, fever, liver disease, urinary tract disease, and skin disease. ${ }^{2}$ Currently, curcumin is thought to be a promising medicinal spice for treating people with Alzheimer's Disease (AD) as it reduces pathology in transgenic mouse models of $\mathrm{AD} .^{3}$

In 2015 , nearly 46.8 million people were living with dementia worldwide. ${ }^{4}$ Approximately, 9.9 million new cases of dementia are recorded each year globally with one new case every 3.2 seconds (ADI, 2017). Around $1.1 \%$ of global GDP (US $\$ 818$ billion) was contributing to the cost of dementia in $2015 .{ }^{5}$ In Australia, dementia is currently the leading cause of disability for people over age of 65 years, and it is the second leading cause of death. ${ }^{6}$ Given the lack of current treatment, investigation of any therapy which may alleviate symptoms or delay deterioration within $\mathrm{AD}$ patients should be conducted.

\section{Mechanisms of action}

The yellow pigmented portion of curcuminoids, which consist of $2-5 \%$ of rhizomes, are comprised of sesquiterpenes, carbohydrates, protein, resins and caffeic acid. ${ }^{7}$ Three major curcuminoids occur naturally in these Curcuma species: Curcumin, demethoxycurcumin and bisdemethoxycurcumin curcumin. ${ }^{7}$ Curcumin phytochemicals, which give the yellow to orange colour to turmeric are thought to be responsible for its therapeutic effects. Commercially available preparations of curcumin (e.g., cavacurcumin, wagner bio-curcumin, longvida, etc.) contain approximately $77 \%$ diferolymethane, $18 \%$ demethoxycurcumin (DMC) and 5\% bimethoxycurcumin (BDMC). ${ }^{7}$ Curcumin is hydrophobic in nature and soluble in dimethyl sulfoxide, acetone, ethanol, and oils. Maximal absorption is around $420 \mathrm{~nm}^{7}$

Curcumin exerts a range of anti-inflammatory effects throughout the body as it is able to penetrate the central nervous system (Venigella, Gyengesi, \& Muench, 2015). Curcumin has been shown to stimulate the proliferation of embryonic neural progenitor cells, and neurogenesis in the adult hippocampus; this may positively impact neuroplasticity. ${ }^{1}$ Its various anti-inflammatory properties have been shown to enhance amyloid beta $(A \beta)$ phagocytosis by microglial macrophages. ${ }^{1}$ Further, curcumin plays a neuroprotective role and leads to inhibition of $A \beta$ aggregation and deposition. ${ }^{1}$ Disilvestro, et al. ${ }^{8}$ showed that plasma content of $A \beta$ protein, a hallmark of $A D$, was reduced following curcumin supplementation in healthy middle-aged people.

Curcumin has also been shown to reduce oxidative stress, lower cholesterol levels, and inhibit acetylcholinesterase activity. ${ }^{1}$ Hishikawa et al. ${ }^{2}$ showed that among AD patients with behavioural and psychological symptoms of dementia, curcumin can regulate levels of neurotransmitters including norepinephrine, dopamine, and serotonin. $^{2}$ It inhibits Mono-Amine Oxidase (MAO)-A and MAO-B enzymes, which play a role in the breakdown of dopamine and serotonin. Plasma alanine amino transferase, a liver injury marker, also showed reduced activity with curcumin. 


\section{An overview of preclinical studies}

Changes in biochemical parameters have been reported in transgenic $\mathrm{AD}$ model rodent studies. In $\mathrm{AD}$-model transgenic mice, $\mathrm{A} \beta$ deposition and plaque burden was decreased following curcumin treatment. Curcumin inhibited $\mathrm{A} \beta$ induced tau phosphorylation, microglial activation, inflammation and oxidative damage. ${ }^{1}$ Genomic instability levels are reduced among transgenic mouse models of AD due to curcumin. Lim et al. (2001) showed that AD model Tg2576 mice aged 10 months old that were fed a curcumin diet (160 parts per million (ppm)) for 6months resulted in reduced levels of oxidised proteins, the inflammatory cytokine, IL-1 $\beta$, astrocyte marker glial fibrillary acidic protein (GFAD), soluble and insoluble $A \beta$, and also plaque burden. Frautschy et al. ${ }^{3}$ also conducted a curcumin study using Sprague-Dawly rats infused with $A \beta 40$ and $A \beta 42$ showing signs of neurodegeneration. Those authors observed that dietary curcumin (2000ppm $(5.43 \mu \mathrm{mol} / \mathrm{g}))$ suppressed $A \beta$-induced oxidative damage and memory impairment, and increased microglial labelling within areas adjacent to $A \beta$ deposits.

Positive effects of curcumin have also been found in behavioural studies on transgenic AD model rodents. Curcumin reversed changes in synaptophysin and post synaptic density 95 (PSD-95) levels related with brain plasticity, and improved rat performance in length and latency within the water maze test. Belviranli et al. showed that aged female rats supplemented with curcumin for $12 \mathrm{~d}$ showed improved spatial memory (Morris water maze test) and reduced oxidative damage was observed in their brains. Han-Chang et al. ${ }^{9}$ showed that oxidative stress was decreased and active avoidance and locomotor activity was improved in the curcumin-protected group in $\mathrm{AD}$ rat model.

McClure et al. ${ }^{10}$ suggested that curcumin can only be applied to animal models as the iv-injection formulation is unfit for human use. A novel technique adopted the use of aerosolite, a curcumin derivative $\mathrm{FMeC} 1$ to facilitate safe delivery to the brain. A translational approach was adopted in a 5XFAD mouse model study which suggested inhalation exposure to an aerosolised $\mathrm{FMeC} 1$ improved distribution of the compound in the brain. ${ }^{10}$ Aerosol delivery has also been confirmed by immunohistochemistry data. $\mathrm{FMeC} 1$ binds to amyloid plaques deposited in hippocampal areas and the cortex. Preclinical studies support the use of aerosolite among humans as it is non-invasive as effective method to improve cognition, but this has not been tested yet. ${ }^{10}$

Other in vivo studies using $A \beta$ infused male Sprague Dawley rat model of $\mathrm{AD}$ examined the effect of combined curcuminoids and individual curcumin constituents with respect to genes related to synaptic plasticity. ${ }^{1}$ Genes affecting actin, Ca- calmodulin-dependent protein kinase formulation (particulate size $<80 \mathrm{~nm}$ ) were studied in vivo using $A D$ transgenic mouse models. Significant improvements in working and cued memory have been observed with the use of noncapsulated curcumin compared to placebo with higher concentration of nano-formulation in plasma and within the brain. Goozee et al. ${ }^{1}$ argued that clinical studies of curcumin depict limited effects to date due to relatively low solubility and bioavailability.

\section{Potential clinical efficacy}

Curcumin and turmeric have been shown to play a significant role in increasing detoxifying enzymes, DNA damage prevention, DNA repair, and decreasing mutations and tumor formation. ${ }^{2}$ Curcumin's potential neuroprotective effects demonstrated in the in vitro and in vivo studies outlined in Goozee et al. ${ }^{1}$ especially protection from the loss of functional neurons and synapses, indicate that it has potential for efficacy against neurodegenerative diseases. Studies conducted by Goozee et al. ${ }^{1}$ support curcumin's neuroprotective and cognitive enhancing properties against $\mathrm{AD}$. Curcumin supplementation has been shown to lead to health promotion, with protective effects observed on cardiovascular health, such as increased plasma content of nitric oxide and lowering plasma concentrations of the SICAM molecule, linked to atherosclerosis. ${ }^{8}$ Even low dose of specific curcumin preparation showed numerous health benefits. As outlined above, the potential efficacy of turmeric supports anti-oxidant, anti-inflammatory, anticarcinogenic, anti-fertilizing, anti-bacterial, anti-microbial, antidepressant activities, and anti-neurodegenerative effects. Significant improvement in neurocognition, injury and wound healing have also been observed. ${ }^{7}$

\section{Aims}

The aim of this systematic review was to evaluate evidence from clinical trials on the efficacy of curcumin to improve cognitive function among people with $\mathrm{AD}$. Given that curcumin is thought to positively impact cognitive function among people with $\mathrm{AD}$, and a number of clinical trials have been completed or are currently underway, a systematic review would help to determine if curcumin has efficacy for the treatment of dementia. Such a review would also help to set future guidelines for conducting clinical trials involving the use of curcumin. To the best of our knowledge, all currently published randomised controlled trials are included in this review.

\section{Methods}

\section{Eligibility criteria}

Eligibility criteria of articles were determined on the basis of several initial scoping searches and reviewing article content. Eligibility criteria were decided in alignment with the research aim, which was to evaluate the efficacy of turmeric for cognitive decline. That is:

- Population: People with dementia (including vascular dementia and $\mathrm{AD}$ ), mild cognitive impairment, cognitive decline

\section{- Intervention: Curcumin treatment}

Peer-reviewed studies were included if they reported a clinical trial and both curcumin intervention and a population of people with cognitive decline. Reviews, cohort studies, annual meetings, conference proceedings, book chapters, case studies, cross sectional studies, preclinical (in vitro and in vivo), and news article collections were excluded, as they did not meet inclusion/exclusion criteria.

\section{Search strategy}

The research team and an experienced librarian reviewed the search strategy before the systematic search was conducted. Peerreviewed journals were searched for using four databases. Abstracts were reviewed from PubMed Central, Science Direct, CINAHL, and PsycINFO ranging from databases' dates of initiation to June 23, 2016. Similar search strategies were carried out in the other three databases, with only minor alteration to allow changes in the use of searching symbols. Reference lists of major articles were also searched for other potential studies.

\section{Data extraction}

Each full text article was assessed for extraction of study characteristics. Data extracted included authors, publication date, 
aim, study design, disease type, aspects of the study population, number of participants, age (mean and SD), gender ratio, recruitment of participants, diagnostic criteria, efficacy measures (mood and quality of life measures, clinical assessments, biochemical measures, cognitive measures or neuropsychological test battery), definition and dosage of curcumin and placebo, intervention administration and duration, methodologies, follow-up period, adverse events and key findings.

\section{Results}

\section{Study selection}

Figure 1 illustrates the study selection process. Five studies met the inclusion criteria for review: Baum et al. ${ }^{11}$ Cox, et al., ${ }^{12}$ Lee et al., ${ }^{13}$
Rainey- Smith et al., ${ }^{14}$ and Ringman et al. ${ }^{3}$ All studies included in the review were unique studies.

\section{Study characteristics}

Table 1 details a summary of the characteristics of the 5 studies including aims, setting, population, intervention type and duration, outcome measures, efficacy of intervention, adverse events, withdrawals, and retention. Two studies included 1 intervention and 1 placebo group. ${ }^{11,14}$ Three studies had parallel treatment arms; of which two studies had two parallel treatment arms $\mathrm{s}^{3,11}$ and one had four parallel treatment arms. ${ }^{13}$ Baum et al. ${ }^{11}$ was carried out in China, Cox et al. ${ }^{12}$ and Rainey-Smith et al. ${ }^{14}$ in Australia, Lee et al. ${ }^{13}$ in Taiwan, and Ringman et al. ${ }^{3}$ in the United States (Figure 1) (Table 1).

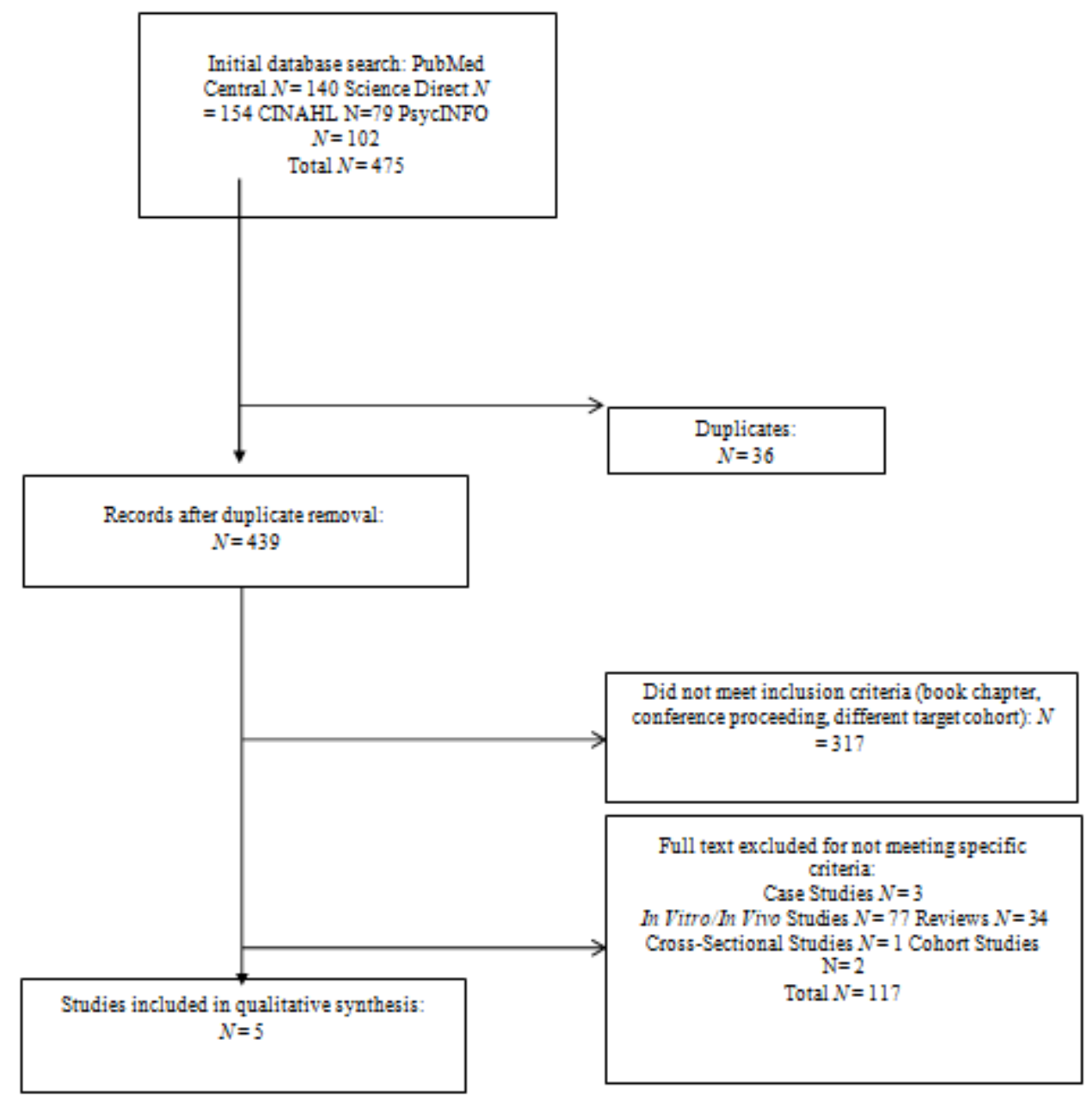

Figure I Flowchart of study selection

Participants: Across all the included studies, the total sample size was $\mathrm{N}=261$ (92 males, 169 females, mean age $=71.8, \mathrm{SD}=6.6$ years) A priori power calculations were carried out to determine sample size in all 5 studies, all of which achieved target sample size. ${ }^{11-14}$ All randomised controlled trials were conducted with Curcuma longa in a human cohort. Across their studies, Baum et al. ${ }^{11}$ and Ringman et al. ${ }^{3}$ examined 70 patients with AD. Lee et al. ${ }^{13}$ studied 48 patients with newly diagnosed untreated pre-diabetes. Rainey Smith et al. (2016) and Cox et al. (2015) observed 156 cognitively healthy older adults altogether.
Recruitment: Only three studies specified recruitment location. They were as follows: Lee et al. ${ }^{13}$ recruited patients from a hospital outpatient clinic, Ringman et al. ${ }^{3}$ recruited from an AD research centre and Rainey Smith et al. ${ }^{14}$ recruited from community dwellings.

Intervention design: Studies examined treatment duration ranging from 1 day to 12 months. All five studies administered an oral intervention. In three studies, oral intervention was in the form of capsule (Cox et al., 2015; Rainey Smith et al., 2016; Ringman et al., 2012). Ringman et al. (2012) administered $500 \mathrm{mg}$ curcumin (or placebo) in 4 capsules twice a day with a fatty meal. Rainey Smith et 
al. (2016) administered one $500 \mathrm{mg}$ BCM-95*CG (BiocurcumaxTM) capsule or placebo three times a day after meals with water. Cox et al. (2015) administered one $400 \mathrm{mg}$ LongVida ${ }^{\circledR}$ Optimised Curcumin capsule once daily (approximately $80 \mathrm{mg}$ curcumin in a solid lipid formulation) or placebo. Baum et al. (2008) administered $4 \mathrm{~g}$ curcumin capsules per day orally in treatment group. In Lee et al. (2014), patients ingested white bread along with either placebo, $2 \mathrm{~g}$ cinnamon, $1 \mathrm{~g}$ turmeric, or cinnamon $2 \mathrm{~g}+$ turmeric $1 \mathrm{~g}$.

Outcome measures: A variety of outcome measures were analysed across the studies: biochemical markers, cognitive/ neuropsychological, and pharmacological. Four studies included biochemical markers as an outcome measure (Cox et al., 2015; Lee et al., 2014; Rainey Smith et al., 2016; Ringman et al., 2012). Four studies analysed neuropsychological measures (Baum et al., 2008; Cox et al., 2015; Lee et al., 2014; Rainey Smith et al., 2016). One study also conducted pharmacokinetic analyses (Baum et al., 2008).

Compliance, withdrawals, and adverse events: Two studies reported on compliance (Cox et al., 2015; Rainey Smith et al., 2016), two reported on withdrawals (Baum et al., 2008; Ringman et al., 2012), and two reported on adverse events (Rainey Smith et al., 2016; Ringman et al., 2012). Information on the reporting of compliance, withdrawals and adverse events is summarised in Table 1.

\section{Efficacy Measures}

Biomarkers: In Baum et al. (2008), Serum A $\square 40$ levels, plasma isoprostanes and Vitamin E were studied as biochemical measures. Serum A $\square 40$ levels did not change among dose of $4 \mathrm{~g}$ /day curcumin at 0,1 , or 6 months among probable AD patients, compared to placebo. No change was observed in plasma isoprostanes levels between baseline and 6 months for this treatment group compared to placebo. Baum et al. (2008) also found vitamin E levels increased during 1 month by $1 \pm 3 \%$ with curcumin $(8 \pm 2 \%$ for capsules vs. $4 \pm 5 \%$ for powder vs. $-21 \pm 5 \%$ with $0 \mathrm{~g}$ placebo) in probable $\mathrm{AD}$ patients. Plasma A $\beta 1-41$, CSF A $\beta 1-42$, CSFtau, ptau, F2 Isoprostanes were studied as biomarker in Ringman et al. (2012), which did not change throughout the study by taking $4 * 500 \mathrm{mg}$ capsules twice daily for 48 weeks with fatty meal placebo or curcumin in patients with mild to moderate $A D$.

Neuropsychological assessment: In Lee et al. ${ }^{13}$ Working Memory (WM) was studied as a cognitive measure (test not specified) which did not change with response to turmeric and cinnamon in newly diagnosed untreated pre-diabetes on cinnamon $2 \mathrm{~g}+$ turmeric $1 \mathrm{~g}$ for 6 hours compared to placebo, cinnamon $2 \mathrm{~g}$, and turmeric $1 \mathrm{~g}$ group. In Ringman et al., ${ }^{3}$ no change was observed in treatment group in patients with mild to moderate AD with respect to Alzheimer's Disease Assessment Scale-Cognitive Subscale (ADAS-cog), Neuropsychiatric Inventory (NPI), Alzheimer's Disease Cooperative Study- Activities of Daily Living (ADCS-ADL) scale or Mini Mental State Examination (MMSE) scores using repeated measures on treatment of $4 * 500 \mathrm{mg}$ capsules twice daily with fatty meal curcumin or placebo for 48 weeks. In Rainey Smith et al. ${ }^{14}$ mean Montreal Cognitive Assessment score improved by 0.64 points in the curcumin group of $1 * 500 \mathrm{mg}$ BCM95*CG (BiocurcumaxTM) capsules three times a day after meals with water and by 0.09 point in the placebo group from baseline to 12 months in cognitively healthy older adults $(\mathrm{N}=96)$. In Cox et al., ${ }^{12} 1 \mathrm{hr}$ after dose of 400mg Long Vida ${ }^{\circledR}$ Optimized Curcumin capsule (once daily administration) there was a significant beneficial effect of acute curcumin treatment on number of correct responses during serial three subtraction task among cognitively healthy older adults for 4 weeks compared to placebo.
Pharmacological measures: Baum et al. ${ }^{11}$ studied pharmacokinetic measures and found increase in plasma levels of curcumin and curcuminoids on $1 * 4 \mathrm{~g}$ curcumin capsules per day orally among patients with probable AD.

\section{Discussion}

The systematic review analysed the efficacy of curcumin for the treatment of dementia, and MCI. Randomised controlled trials incorporating curcumin treatment were evaluated. The discussion will focus on the two aims of this review (1) intervention efficacy and (2) safety measures.

\section{Study characteristics}

The majority of studies tested curcumin intervention, with most using a tablet or capsule for oral administration. Details regarding the composition of commercially available extracts were not found among most studies such as Ringman et al. ${ }^{3}$ Treatment duration varied considerably between studies from 1 day to 12 months which added complexity to comparisons between studies. Different methodologies such as questionnaires and pharmacological analytic methods were used which also made results difficult to compare.

\section{Intervention efficacy}

Outcome measures including biomarker, neuropsychological and pharmacological measures were analysed. There were no significant changes among major biomarker measures. No change was observed among biomarkers such as Serum A $\beta 40$, plasma isoprostanes, plasma A $\beta 1-41$, CSF A $\beta 1-42$, CSFtau, ptau, and F2 Isoprostanes. ${ }^{3,11}$ Vitamin E levels showed significant improvement after curcumin treatment among probable AD patients. ${ }^{11}$ There was no change observed among cognitive and quality of life measures such as working memory, ADAS- Cog, NPI, ADCS-ADL, and MMSE when compared to placebo. ${ }^{3}$ Mean MoCA score showed improvement and beneficial effect was observed on number of correct responses during serial three subtraction task. ${ }^{14}$ Pharmocokinetic measures such as plasma levels of curcumin and curcuminoids increased significantly. ${ }^{11}$

\section{Comparison to preclinical studies}

Results from clinical trials are not consistent with findings from preclinical studies. This section will compare the findings on curcumin between clinical and preclinical studies. Curcumin exhibited various positive outcomes in animal models such as neuroprotective effects against AD, Parkinson's disease, epilepsy and depression. ${ }^{2}$ In transgenic mice, curcumin shows protective effects, such as disruption of $\beta$-amyloid deposition and $\beta$-amyloid disaggregation, preventing oligomer and fibril formation; even at low dose of curcumin administration. ${ }^{15}$ Curcumin seems to be more effective when treating transgenic animal models than in sporadic late-onset AD in humans with associated cerebral atrophy and neuronal death. Some positive outcomes of curcumin have been observed in a few of the clinical trials explored here, but the evidence is not convincing enough to draw any conclusions in relation to efficacy. ${ }^{14}$

Some of the lowest prevalence rates of $\mathrm{AD}$ have been observed in India, where there is widespread consumption of turmeric. ${ }^{2}$ Associations have been found between curry consumption and better cognitive function in elderly Asian community- dwellers. ${ }^{16}$ However, in $\mathrm{AD}$ clinical trials, curcumin has been unable to produce sufficient brain levels of curcumin following oral administration. ${ }^{15}$ Hence, it is difficult to understand the beneficial effect on cognition observed in cohort studies. On considering heterogeneous populations such 
as dementia and probable $\mathrm{AD}$, curcumin supplementation shows no effect on serum total cholesterol, LDL-C, triglycerides and HDL-C. ${ }^{17}$ There is not sufficient evidence from clinical trials to suggest that curcumin could be an effective treatment for dementia. ${ }^{15}$ Curcumin may be effective for other indications, as it has been found to be effective and safe agent in treatment in patients with major depressive disorder without concurrent suicide ideation or other psychotic disorders. ${ }^{18}$

\section{Safety measures}

Curcumin short term use appears to be safe. The majority of studies reported compliance, withdrawals and adverse events., ${ }^{3,11-14}$ Patients withdrew due to gastrointestinal complaints, falls, dizziness, respiratory tract infection, worsened memory and difficulty swallowing pills. Haematological safety of using curcumin treatment was found among all clinical trials reviewed here. No other major side effects were reported. This is probably due to curcumin having poor water solubility and poor oral bioavailability, with administered doses primarily excreted in faeces. ${ }^{2}$ To overcome this challenge, new formulations along with piperidine, nanoparticle, micelles and liposomes have been developed to improve bioavailability. ${ }^{15}$ Major focus in relation to its dose response is to increase the bioavailability, BBB penetration and to sustain the half-life of curcumin. ${ }^{1}$

\section{Strengths, limitations, and recommendations}

Heterogeneity of the intervention and methodology employed made meta-analysis difficult. Outcome measures were difficult to analyse due to variation in methodology. Two studies did not report recruitment setting. ${ }^{11,12}$ Short follow up periods makes it difficult to detect potential long-term changes in dementia symptoms and progression. Reliable findings could be yielded from current ongoing clinical trials as they have larger sample sizes. Future work could consider focusing on only one intervention or methodology in order to quantify efficacy such as blood biomarkers or neuroimaging. Curcumin's poor bioavailability is a major hurdle. Hence, as discussed, formulation along with piperidine, nanoparticle, micelles and liposomes should be developed and utilised in future research. ${ }^{15,19-27}$

\section{Conclusion}

This systematic review summarised and critically appraised efficacy of curcumin treatment on people with MCI and dementia. It was found that most studies focused on people with $\mathrm{AD}$, utilized curcumin intervention that was on average 6 months long and used biomarker, neuropsychological and pharmacological assessment as an outcome measure. Nearly all studies reported positive results. According to pharmacodynamics, curcumin acts as neuroprotective against dementia than reversal medication. To the date this review was finalized (15 May, 2017) only few clinical trials have been conducted which have led to inconclusive results. Future findings from ongoing clinical trials will explain potential therapeutic efficacy of curcumin for dementia in more detail.

\section{Acknowledgements}

None.

\section{Conflicts of interest}

Author declares that there are no conflicts of interest.

\section{Funding}

None.

\section{References}

1. Goozee KG, Shah TM, Sohrabi HR, et al. Examining the potential clinical value of curcumin in the prevention and diagnosis of Alzheimer's disease. Br J Nutr. 2016;115(3):449-465.

2. Hishikawa N, Takahashi Y, Amakusa Y, et al. Effects of turmeric on Alzheimer's disease with behavioral and psychological symptoms of dementia. Ayu. 2012;33(4):499-504.

3. Ringman JM, Frautschy SA, Teng E, et al. Oral curcumin for Alzheimer's disease: tolerability and efficacy in a 24-week randomized, double blind, placebo-controlled study. Alzheimers Res Ther. 2012;4(5):43.

4. Alzheimer's Disease International. Dementia statistics. UK: Alzheimer's disease International. 2017.

5. Wimo A, Guerchet M, Ali GC, et al. The worldwide costs of dementia 2015 and comparisons with 2010. Alzheimers Dement. 2017;13(1):1-7.

6. Australian Institute of Health and Welfare. Dementia in Australia $(A I H W)$. Australia: Australian Institute of Health and Welfare; 2012. p. 240

7. Aggrawal BB, Kunnumakkara AB. Molecular Targets and Therapeutic Uses of Spices; Modern Uses for Ancient Medicine. USA: World Scientific; 2009. p. 456

8. DiSilvestro RA, Joseph E, Zhao S, et al. Diverse effects of a low dose supplement of lipidated curcumin in healthy middle aged people. Nutr J. 2012;11:79.

9. Han-Chang H, Bo-Wen Z, Yu G, et al. Antioxidative and Neuroprotective Effects of Curcumin in an Alzheimer's Disease Rat Model Co-Treated with Intracerebroventricular Streptozotocin and Subcutaneous DGalactose. Journal of Alzheimer's Disease. 2016;52(3):899-911.

10. McClure R, Yanagisawa D, Stec D, et al. Inhalable curcumin: offering the potential for translation to imaging and treatment of Alzheimer's disease. J Alzheimers Dis. 2015;44(1):283-295.

11. Baum L, Lam CWK, Cheung SKK, et al. Six-month randomized, placebo-controlled, double-blind, pilot clinical trial of curcumin in patients with Alzheimer disease. $J$ Clin Psychopharmacol. 2008;28(1):110-113.

12. Cox KHM, Pipingas A, Scholey AB. Investigation of the effects of solid lipid curcumin on cognition and mood in a healthy older population. $J$ Psychopharmacol. 2015;29(5):642-651.

13. Lee MS, Wahlqvist ML, Chou YC, et al. Turmeric improves postprandial working memory in pre-diabetes independent of insulin. Asia Pac J Clin Nutr. 2014;23(4):581-591.

14. Rainey-Smith SR, Brown BM, Sohrabi HR, et al. Curcumin and cognition: a randomised, placebo-controlled, double-blind study of community-dwelling older adults. Br J Nutr. 2016;115(12):2106-2113.

15. Brondino N, Re S, Boldrini A, et al. Curcumin as a therapeutic agent in dementia: a mini systematic review of human studies. Scientific World Journal. 2014;2014:174282.

16. $\mathrm{Ng} \mathrm{TP}$, Chiam PC, Lee $\mathrm{T}$, et al. Curry consumption and cognitive function in the elderly. Am J Epidemiol. 2006;164(9):898-906.

17. Sahebkar A. A systematic review and meta-analysis of randomized controlled trials investigating the effects of curcumin on blood lipid levels. Clin Nutr. 2014;33(3):406-414.

18. Sanmukhani J, Satodia V, Trivedi J, et al. Efficacy and safety of curcumin in major depressive disorder: a randomized controlled trial. Phytother Res. 2014;28(4):579-585.

19. Yang F, Lim GP, Begum AN, et al. Curcumin inhibits formation of amyloid $\beta$ oligomers and fibrils, binds plaques, and reduces amyloid in vivo. J Biol Chem. 2005;280(7):5892-5901. 
20. Zhang DW, Fu M, Gao SH, et al. Curcumin and diabetes: a systematic review. Evidence-Based Complementary and Alternative Medicine. 2013.2013:636053.

21. Zhang ZG, Niu XY, Lu AP, et al. Effect of curcumin on aged Drosophila Melanogaster: A pathway prediction analysis. Chin J Integr Med. 2015;21(2):115-22.

22. Potter PE. Curcumin: a natural substance with potential efficacy in Alzheimer's disease. J Exp Pharmacol. 2013;5:23-31.

23. Gota VS, Maru GB, Soni TG, et al. Safety and pharmacokinetics of a solid lipid curcumin particle formulation in osteosarcoma patients and healthy volunteers. J Agric Food Chem. 2010;58(4):2095-2099.
24. Gupta SC, Patchva S, Aggarwal BB. Therapeutic roles of curcumin: lessons learned from clinical trials. AAPS J. 2013;15(1):195-218.

25. Ahmad K, Ansari VA, Singh K, et al. Curcuma Longa: boon for health care system with its biomedical application. International Journal of Pharmaceutical Sciences and Research. 2015;6(10):4168-4173.

26. Ahmed T, Gilani AH. Therapeutic Potential of Turmeric in Alzheimer's Disease: Curcumin or Curcuminoids? Phytother Res. 2014;28(4):517525 .

27. Ashraf K, Mujeeb M, Ahmad A, et al. Determination of Curcuminoids in Curcuma longa Linn. by UPLC/Q-TOF-MS: An Application in Turmeric Cultivation. J Chromatogr Sci. 2015;53(8):1346-1352. 